\title{
Elucidation of the Heat-Flux Limit from Magnetic-Island Heating
}

\author{
M.Z. Tokar and A. Gupta \\ Institut für Energieforschung-Plasmaphysik, Forschungszentrum Jülich, Association EURATOM-FZJ, \\ Trilateral Euregio Cluster, Germany* \\ (Received 26 April 2007; revised manuscript received 30 August 2007; published 28 November 2007)
}

\begin{abstract}
Recent experiments on heating of magnetic islands in a tokamak are analyzed to assess plasma transport characteristics. By comparing with the experimental data, both perpendicular and parallel components of the electron heat conductivity in the island are determined. As a consequence, the so-called heat-flux limit factor $\xi$, the ratio of the parallel heat conduction flux in a collisionless plasma to that transferred by freestreaming electrons, can be estimated. The found factor $\xi$ does not contradict that established earlier by interpreting laser plasma experiments.
\end{abstract}

DOI: 10.1103/PhysRevLett.99.225001

PACS numbers: 52.35.Py, 52.55.Fa

Introduction.-Diverse MHD instabilities such as ideal ballooning, peeling, tearing modes, break closed magnetic surfaces and destroy the axis symmetry of magnetic configuration in a tokamak. The resulting powerful transport along magnetic field lines can contribute substantially to particle and heat losses from the plasma and significantly affect confinement as this takes place by the development of neoclassical tearing modes in the core [1] and edge localized modes (ELM) at the plasma periphery [2]. Therefore the transport characteristics parallel to the magnetic field, in particular the parallel heat conduction $\kappa_{\|}$, are of paramount importance for comprehension and a quantitative description of such phenomena. In particular, it has been demonstrated recently that both the intensity and the collisionality dependence of energy losses by the type I ELMs are governed essentially by $\kappa_{\|}$[3]. It is also relevant for the mechanisms of ELM mitigation with external magnetic field perturbations [4].

In plasmas, the dominant contribution to $\kappa_{\|}$is due to light electrons. If electrons collide with each other often enough, the Spitzer-Härm formula can be adopted [5]: $\kappa_{\|} \approx \kappa_{\|}^{\mathrm{SH}}=3.16 n V_{\mathrm{th}} \lambda$, with $n, V_{\mathrm{th}}=\sqrt{T / m}$ and $\lambda$ being the density, thermal velocity, and collision mean free path length of electrons, $T$ and $m$ - their temperature and mass, respectively. With dwindling collisionality this approximation becomes inadequate when $\lambda$ exceeds some per mill of the characteristic dimension for the temperature change, $L_{T}=1 /\left|\nabla_{\|} \ln T\right|$, i.e., much earlier than the fluid approximation breaks down. The reason for this: heat carrying electrons with velocities by a factor of 3-4 larger than $V_{\text {th }}$ get collisionless [6]. The significance of these suprathermal particles for the heat conduction process manifests also in the fact that contradictory to naive expectations, the free-streaming heat flux, $\xi n V_{\mathrm{th}} T$ with $\xi \approx 1$, fails to reproduce the conductive heat transfer in a collisionless case. This was first demonstrated by interpreting laser fusion experiments. These studies [7] have elucidated that a significant heat-flux limitation has to be introduced by assuming $0.03 \leq \xi \leq 0.1$. Fokker-Planck simulations of laser produced plasmas [8] and a tokamak scrape-off layer with strong recycling [6] have assured this limitation emanating due to nonlocal effects. These effects diminish the perturbation in the distribution function caused by the temperature gradient and being responsible for the heat conduction process [9].

Up to now there has been an absence of experimental evidence for the existence of the heat-flux limit in tokamak plasmas. In this Letter we analyze from this point of view recent experiments on the tokamak TEXTOR where a tearing mode with the poloidal and toroidal wave numbers $M$ and $N$ equal to 2 and 1, respectively, was triggered by the dynamic ergodic divertor (DED) [10]. The arising magnetic islands were heated by electron cyclotron resonance heating (ECRH) [11] and detailed measurements of the electron temperature profile in islands have been performed with the 2D electron cyclotron emission imaging (ECEI) diagnostic [12]. By interpreting these results the heat conduction components, both perpendicular and parallel to the magnetic field, $\kappa_{\perp}$ and $\kappa_{\|}$, respectively, are determined. The elucidated heat-flux limit factor $\xi$ is at the low edge of the range established earlier in laser plasma experiments [7].

Heat transport analysis. - Heretofore heat transport in configurations with magnetic islands has been analyzed analytically and numerically by neglecting heat sources inside islands [13-16]. In this Letter we take these into account by applying analytical approaches. The most rigorous one has been developed in Ref. [14] by considering two limit cases of "small" and "large" islands where the island width $w$ is either much smaller or much larger than the critical one $w_{c}$. The latter is controlled by the ratio of $\kappa_{\perp}$ and $\kappa_{\|}$, parameters which have to be ascertained in this study. Thus, one cannot say a priori which limit is relevant. Therefore the theory proposed in Ref. [14] will be generalized here for both limit cases by including the plasma heating in the magnetic island. For this purpose we take into account that the total ECRH power has been launched into a layer centered on the resonant surface (RS), being much thinner than the island width. Owing to the island 
rotation the power is distributed nearly homogeneously over the RS. Therefore it is assumed that the heat deposition layer is a infinitesimally thin toroidal shell centered on the RS. The deposited heat flows from the RS towards both the plasma core and edge with the heat-flux density $q_{\mathrm{rs}}=$ $P_{\mathrm{ECRH}} /\left(8 \pi^{2} r_{\mathrm{rs}} R_{\mathrm{rs}}\right)$, where $P_{\mathrm{ECRH}}$ is the heating power, $r_{\mathrm{rs}}$ and $R_{\mathrm{rs}}$ are the minor and major radii of the RS. Since the Ohmic power deposited in the plasma core is transported to the plasma edge mainly through regions close to the $X$ point of islands [14] it does not change strongly the temperature perturbation produced by the island heating.

By applying the ECRH the temperature in the island $O$ point rises quickly in roughly $10 \mathrm{~ms}$ by a maximal $25 \%$ and remains nearly constant during the heating phase lasting up to $700 \mathrm{~ms}$ [12]. Thus the temperature profile in the island can be described by a stationary heat transport equation [14]. By including the heat source this equation can be conveniently written in the form

$$
\left(\sin \zeta \frac{\partial}{\partial y}+4 y \frac{\partial}{\partial \zeta}\right) g\left(\sin \zeta \frac{\partial \tilde{T}}{\partial y}+4 y \frac{\partial \tilde{T}}{\partial \zeta}\right)+\sigma \frac{\partial^{2} \tilde{T}}{\partial y^{2}}=-\frac{w^{2} Q}{4 \kappa_{\|} b_{r}^{2}},
$$

where $\tilde{T}$ is the temperature perturbation due to the presence of the island and heating; $\zeta \equiv M \vartheta-N \varphi$ the phase angle of the radial magnetic field perturbation, $B_{r}=B_{r}^{0} \sin \zeta$, with $\vartheta$ and $\varphi$ being the poloidal and toroidal angles, respectively. As in Ref. [14] the island $O$ point is located at $\zeta=\pi ; y=2\left(r-r_{\mathrm{rs}}\right) / w$ is the distance from the RS measured in the island half-widths; $\sigma=\kappa_{\perp} /\left(\kappa_{\|} b_{r}^{2}\right)$ with $b_{r}=B_{r}^{0} / B$ and $B$ being the total magnetic field, i.e., nearly its toroidal component; the metric coefficient $g=$ $1 / \sqrt{1+\left(\frac{8 r}{M w} \frac{y}{\sin \zeta}\right)^{2}}$ accounts for the variation of the distance between magnetic surfaces with $\zeta$ and $y ; Q$ is the heating power density. By taking into account the heating source localization at the RS, the temperature perturbation out of the RS is described by Eq. (1) with zero right-hand side. This is similar to Eq. (24) in Ref. [14] where the critical island width $w_{c}=w(\sigma / 4)^{1 / 4}$ and radial coordinate $X=$ $2 w y / w_{c}$ have been used. The heating at the RS defines the boundary condition for $\tilde{T}$ at $y=0$. This can be found by integrating Eq. (1) over the infinitesimally thin heating layer or, in a more transparent way, from the heat-flux continuity at the border of this layer, $q_{\mathrm{rs}}=-\kappa_{\perp} \partial \tilde{T} / \partial r+$ $q_{\|}^{r}$. The former contribution on the right-hand side is due to perpendicular heat conduction and the latter one-the radial component of the heat flux along magnetic field lines

$$
q_{\|}^{r}=-\frac{B_{r}}{B} \kappa_{\|} \nabla_{\|} \tilde{T}=-\kappa_{\|} b_{r}^{2} \sin ^{2} \zeta \frac{\partial \tilde{T}}{\partial r} .
$$

As a result one has

$$
\frac{\partial \tilde{T}}{\partial y}(0)=-\frac{w q_{\mathrm{rs}}}{2 \kappa_{\|} b_{r}^{2}\left(\sigma+\sin ^{2} \zeta\right)} .
$$

Small island limit. - In this approximation, corresponding to $w \ll w_{c}$ or $1 \ll \sigma$, the temperature perturbation is primarily just a function of $y$. This contradicts to the experimental observations [12] clearly exhibiting a significant $\zeta$ - dependence in $T$. Therefore one can reasonably expect that this limit is not realized. However, for the completeness of our analysis we consider it. As in Ref. [14] it is assumed $\tilde{T}(y, \zeta)=\tilde{T}_{0}(y)+\tilde{T}_{1}(y) \cos \zeta$ and the functions $\tilde{T}_{0}(y)$ and $\tilde{T}_{1}(y)$ are governed by Eqs. (29) of Ref. [14]. However, their boundary conditions at the RS are modified. From Eq. (3) one gets $\frac{\partial \tilde{T}}{\partial y}(0) \approx-\frac{w q_{\mathrm{rs}}}{2 \kappa_{\perp}}\left(1-\frac{1}{2 \sigma}+\right.$ $\left.\frac{\cos 2 \zeta}{2 \sigma}\right)$ and

$$
\frac{d \tilde{T}_{0}}{d y}(0) \approx-\frac{w q_{\mathrm{rs}}}{2 \kappa_{\perp}}, \quad \frac{d \tilde{T}_{1}}{d y}(0) \approx 0 .
$$

Thus, in the case in question the function $f$, controlling the $y(X)$ dependence of $\tilde{T}_{1}$, see Eq. (32) in Ref. [14], satisfies the boundary condition $d f / d y(0)=0$. Numerical solution of Eq. (33) from Ref. [14] with this boundary condition gives $f(0)=2.5$ instead of $f(0)=0$ adopted there.

ECEI diagnostics [12] allows apparent identification of the poloidal cross sections of isothermal surfaces in islands. These surfaces are characterized by the radial and poloidal half-widths $x_{*}$ and $\vartheta_{*}$, respectively, corresponding to the phase coordinates $y_{*}=2 x_{*} / w$ and $\zeta_{*}=\pi+M \vartheta_{*}$ $\left(\varphi_{*}\right.$ is constant for poloidal cross sections of isotherms and is assumed equal to zero). The radial temperature difference $\quad \delta_{r} \tilde{T} \approx \tilde{T}(0, \pi)-\tilde{T}\left(y_{*}, \pi\right) \approx-y_{*} \partial \tilde{T}_{0} / \partial y(0)=$ $q_{\mathrm{rs}} x_{*} / \kappa_{\perp}$. With $\delta_{r} \tilde{T}, q_{\mathrm{rs}}$ and $x_{*}$ known from the experiment one can compute the perpendicular heat conduction

$$
\kappa_{\perp} \approx q_{\mathrm{rs}} x_{*} / \delta \tilde{T}_{r} .
$$

Moreover, on isotherms $\delta_{r} \tilde{T}$ is equal to $\tilde{T}(0, \pi)-$ $\tilde{T}\left(0, \zeta_{*}\right) \approx-\tilde{T}_{1}(0)\left(1+\cos \zeta_{*}\right)$ and this results in

$$
\sigma=\left[\frac{f(0)\left(1+\cos \zeta_{*}\right) w}{8 \sqrt{2} x_{*}}\right]^{4} .
$$

As an example we consider the case of $P_{\mathrm{ECRH}}=300 \mathrm{~kW}$ in Ref. [12] and the isotherm with $\delta_{r} \tilde{T} \approx 35 \mathrm{eV}$. This is characterized by $x_{*}=2.7 \mathrm{~cm}$ and $\vartheta_{*}=0.83$. For typical $q_{\mathrm{rs}} \approx 0.7 \mathrm{~W} / \mathrm{cm}^{2}$ and $w=6 \mathrm{~cm}$, one obtains $\kappa_{\perp} \approx$ $3.4 \times 10^{17} \mathrm{~cm}^{-1} \mathrm{~s}^{-1}$ and $\sigma \approx 0.08$. As it was expected, the latter is in contradiction to the small island approximation, $\sigma \gg 1$.

Large island limit.-This limit corresponds to $\sigma<1$ and parallel heat conduction dominating over the perpendicular one and heating. In this case the temperature perturbation has to be, in the first approximation, constant on island magnetic surfaces corresponding to a constant $\Omega=$ $2 y^{2}+\cos \zeta$. Indeed, by assuming $\tilde{T}=T_{0} y^{2}+T_{1} \cos \zeta$, where $T_{0}$ and $T_{1}$ are some constant factors, and substituting this into Eq. (1) where $\kappa_{\|} \rightarrow \infty$ and, thus, $\sigma \rightarrow 0$, one obtains $T_{1}=T_{0} / 2$ and $\tilde{T}=\Omega T_{0} / 2$. By considering the effect of perpendicular heat conduction and heating as 
small, we look for the solution of Eq. (1) in the form $\tilde{T}=$ $\Omega T_{0} / 2+\tilde{T}_{2}$, where $\tilde{T}_{2}(y, \zeta) \approx \Theta(y) \Psi(\zeta)$. The requirement that $\tilde{T}$ has to satisfy the boundary condition (3) at the RS results in

$$
\tilde{T}=\frac{T_{0}}{2} \Omega+\frac{w q_{\mathrm{rs}}}{2 \kappa_{\|} b_{r}^{2}} \frac{\Theta(y)}{\sigma+\sin ^{2} \zeta}
$$

with the following boundary condition for $\Theta(y)$

$$
\frac{d \Theta}{d y}(0)=-1 \text {. }
$$

The equation, governing $\Theta$, is obtained by substituting $\tilde{T}$ from Eq. (7) into Eq. (1) and averaging over the phase angle $\zeta$. This provides

$$
\frac{d}{d y}\left(\gamma \frac{d \Theta}{d y}\right)=-\frac{2 \pi T_{0} \kappa_{\perp}}{w q_{\mathrm{rs}}}
$$

with

$$
\gamma(y)=\int_{0}^{1} \frac{\left(1-s^{2}\right) d s}{\sqrt{\alpha^{2} y^{2}+1-s^{2}}\left(\sigma+1-s^{2}\right)},
$$

where $\alpha=8 r /(M w)$. According to [14] all $\tilde{T}_{\nu \geq 2}$ subside fast with the distance from the island and we assume

$$
\Theta(1)=0
$$

as the second boundary condition for $\Theta$. The integration provides

$$
\Theta=\theta_{0}(y)+\frac{2 \pi T_{0} \kappa_{\perp}}{w q_{\mathrm{rs}}} \theta_{1}(y)
$$

with $\theta_{0}(y)=\int_{y}^{1} \frac{\gamma(0)}{\gamma(y)} d y$ and $\theta_{1}(y)=\int_{y}^{1} \frac{y d y}{\gamma(y)}$ shown in Fig. 1 for different combinations of the parameters $\alpha$ and $\sigma$ characteristic for ECRH of different power levels in TEXTOR [12].

In order to determine the parameters $T_{0}, \kappa_{\perp}$, and $\sigma$ we calculate according to Eqs. (7) and (12) the differences in the temperature perturbation in four points on the RS, $y=$ $0: \Omega_{i=1-4}=-0.8,-0.4,0$, and 0.4 . By equating these to

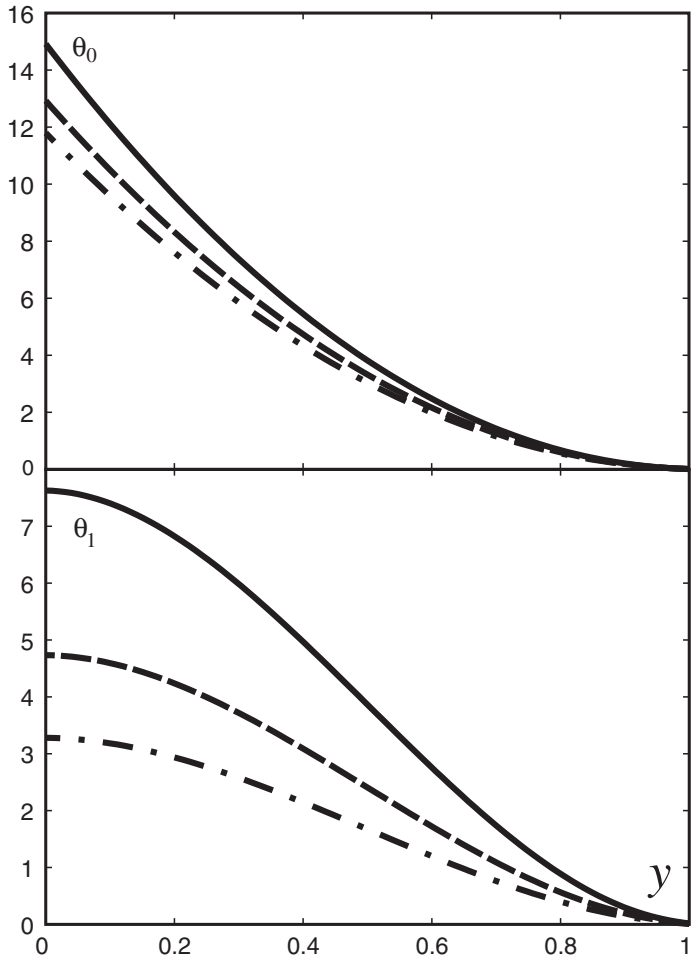

FIG. 1. Functions $\theta_{0}(y)$ and $\theta_{1}(y)$ calculated for $\alpha=24, \sigma=$ 0.5 (solid curves), $\alpha=20, \sigma=0.2$ (dashed curves), and $\alpha=$ $17, \sigma=0.05$ (dash-dotted curves), characteristic for the ECRH experiments in Ref. [12] with heating power of 400, 300, and $200 k W$, respectively.

the experimental values $\Delta_{\Omega_{i}, \Omega_{j}}$ taken from Fig. 4 of Ref. [12], one gets

$$
\begin{gathered}
T_{0}=-2.5 \Delta_{-0.4,0.4}, \\
\sigma=\frac{\Delta_{-0.8,0}-9 / 8 \Delta_{-0.8,0.4}+11 / 16 \Delta_{-0.4,0.4}}{4 / 3 \Delta_{-0.8,0.4}-\Delta_{-0.4,0.4}-\Delta_{-0.8,0}},
\end{gathered}
$$

and

$$
\kappa_{\perp} \approx \frac{0.32 \theta_{0}(0) w q_{\mathrm{rs}}}{\left[\left(\Delta_{-0.8,0}-\Delta_{-0.4,0.4}\right)(\sigma+1.36+0.36 / \sigma)+5 \theta_{0}(1) \Delta_{-0.4,0.4}\right]} .
$$

With the temperature profiles from Ref. [12] one obtains $\sigma$ in the ranges $0-0.1,0.1-0.3$, and $0.5-1$, for the ECRH power of 200,300, and $400 \mathrm{~kW}$, respectively. Thus, the applicability condition for the large island approximation, $\sigma<1$, is fulfilled. The perpendicular heat conduction $\kappa_{\perp}$ is within the range $2.5-3.1 \times 10^{17} \mathrm{~cm}^{-1} \mathrm{~s}^{-1}$, i.e., close to the level found in the small island approximation. One can see, in the case of the temperature perturbation being exactly constant on magnetic surfaces, $\tilde{T} \sim \Omega$, the $\sigma$ and transport coefficients given by Eq. (14) and (15) are undefined. Thus, namely, the deviation from such a constancy provides a possibility to determine $\kappa_{\perp}$ and $\kappa_{\|}$.
Heat-flux limit factor.-The formula interpolating expressions for the heat-flux density in the collision dominated and collisionless limits has been introduced in Ref. [7]. For the heat conduction this provides

$$
\kappa_{\|} \approx \kappa_{\|}^{\mathrm{SH}} /\left(1+\frac{3.16}{\xi} \frac{\lambda}{L_{T}}\right) .
$$

On a particular flux surface the $e$-folding length for the temperature change along field lines, $L_{T}$, can be estimated as $L_{\|} T / \Delta_{\|} T$ with $\Delta_{\|} T$ and $L_{\|}$being the temperature variation along the field line section of the length $L_{\|}$. As 


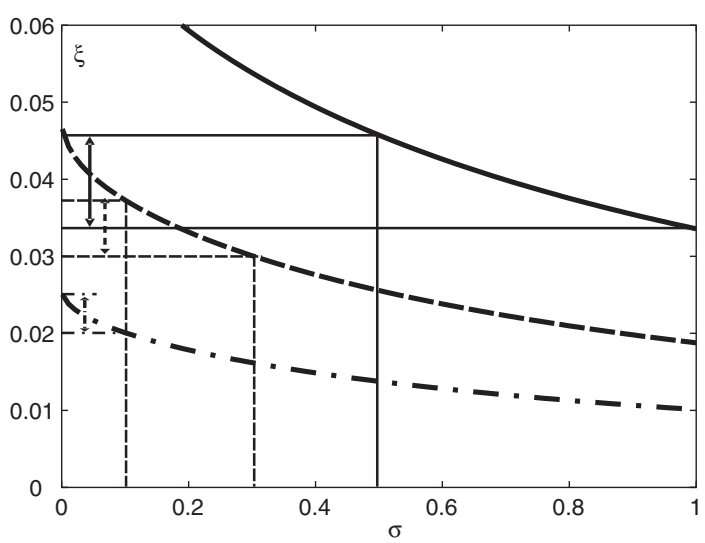

FIG. 2. $\sigma$-dependence of the heat-flux limit $\xi$ calculated according to Eq. (20) for the condition of ECRH in TEXTOR with different power levels of 400 (solid curve), 300 (dashed curve), and $200 \mathrm{~kW}$ (dash-dotted curve). The vertical lines of the same pattern indicate the $\sigma$-ranges determined from the experimental data of Ref. [12] according to Eq. (14); the vertical arrows - the corresponding $\xi$-intervals.

a typical example we consider the surface with $\Omega=0$ and the field line part between the RS and $y=0.5$. In such a case an assessement of $\Delta_{\|} T$ from Eqs. (7) and (12) and Fig. 1 yields

$$
\Delta_{\|} T \approx \frac{\theta_{0}(0)-\theta_{0}(0.5)}{\sigma+1} \frac{w q_{\mathrm{rs}}}{2 \kappa_{\|} b_{r}^{2}} \approx \frac{0.37 \theta_{0}(0)}{\sigma+1} \frac{w q_{\mathrm{rs}}}{\kappa_{\|} b_{r}^{2}}
$$

and for $L_{\|}$one has

$L_{\|}=\int_{r_{\mathrm{rs}}}^{r_{\mathrm{rs}}+0.25 w} \frac{B}{B_{r}} d r=\frac{w}{2 b_{r}} \int_{0}^{0.5} \frac{d y}{\sqrt{1-4 y^{2}}} \approx 0.26 w / b_{r}$.

For collisionless plasmas in question the second term in the brackets in Eq. (16) is much larger than 1. Therefore for the heat-flux limit factor $\xi$ we obtain

$$
\xi \approx \frac{3.16 \kappa_{\|}}{\kappa_{\|}^{\mathrm{SH}}} \frac{\lambda}{L_{T}} \approx \frac{1.42 q_{\mathrm{rs}} \theta_{0}(0)}{(\sigma+1) n V_{\mathrm{th}} T b_{r}} .
$$

The relative amplitude of the radial magnetic field perturbation, $b_{r}$, is estimated from the measured island width $w$ according to the formula [14]: $b_{r}=(w / 4)^{2} \hat{s}_{\mathrm{rs}} N /\left(r_{\mathrm{rs}} R_{\mathrm{rs}}\right)$. At the RS with $r_{\text {rs }} \approx 30 \mathrm{~cm}$ the magnetic shear $\hat{s}_{\text {rs }} \approx 1$ and we obtain $\xi$ with parameters in practical units, see the square brackets

$$
\xi \approx \frac{0.14 \theta_{0}(0)}{\sigma+1} \frac{P_{\mathrm{ECRH}}^{[\mathrm{MW}]}}{n_{\left[10^{13} \mathrm{~cm}^{-3}\right]}^{1.5} T_{[\mathrm{kev}]}^{1.5} w_{[\mathrm{cm}]}^{2}} .
$$

Figure 2 demonstrates the $\sigma$ dependences of $\xi$ computed according to Eq. (20) for different levels of the ECRH power and the temperature profiles on the RS from Fig. 4 of Ref. [12]. The corresponding $\sigma$-intervals and resulting $\xi$-ranges are shown by vertical lines and arrows, respectively. In spite of the broad range of the parameter $\sigma$, the found $\xi$-factor varies in a relatively narrow interval from 0.02 to 0.045 . These values lie at the lower edge of the $\xi$-range found by interpreting laser fusion experiments [7], $0.03 \leq \xi \leq 0.1$, and can be considered as the first experimentally based evidence for the parallel heat-flux limit in tokamak plasmas.

This study has been partly performed within No. GRK1203 of the German Research Society.

*www.fz-juelich.de/ipp

[1] Z. Chang et al., Phys. Rev. Lett. 74, 4663 (1995).

[2] F. Wagner et al., Phys. Rev. Lett. 49, 1408 (1982).

[3] M. Z. Tokar et al., Plasma Phys. Controlled Fusion 49, 395 (2007).

[4] M.Z. Tokar et al., Phys. Rev. Lett. 98, 095001 (2007).

[5] S. I. Braginskii, in Reviews of Plasma Physics edited by M. A. Leontovich (Consultants Bureau, New York, 1965), Vol. 1, p. 205.

[6] R. Chodura, Contrib. Plasma Phys. 32, 219 (1992).

[7] R. C. Malone, R. L. McCrory, and R. L. Morse, Phys. Rev. Lett. 34, 721 (1975).

[8] J. F. Luciani, P. Mora, and J. Virmont, Phys. Rev. Lett. 51, 1664 (1983).

[9] E. M. Epperlein and R. W. Short, Phys. Fluids B 4, 2211 (1992).

[10] R. C. Wolf et al., Nucl. Fusion 45, 1700 (2005).

[11] E. Westerhof et al., Nucl. Fusion 47, 85 (2007).

[12] I. G. J. Classen et al., Phys. Rev. Lett. 98, 035001 (2007).

[13] M.Z. Tokar, 14th European Conference on Controlled Fusion and Plasma Physics, Madrid (European Physical Society, Geneva, 1987), Vol. 13F, Part 2, p. 687.

[14] R. Fitzpatrick, Phys. Plasmas 2, 825 (1995).

[15] Q. Yu, Phys. Plasmas 13, 062310 (2006).

[16] M. Holzl et al., Phys. Plasmas 14, 052501 (2007). 